Article

\title{
Genetic Manipulation of a Lipolytic Yeast Candida aaseri SH14 Using CRISPR-Cas9 System
}

\author{
Zool Hilmi Ibrahim ${ }^{1,2, \dagger}$, Jung-Hoon Bae ${ }^{1, \dagger}$, Sun-Hee Lee ${ }^{1}$, Bong Hyun Sung ${ }^{1,2}$, \\ Ahmad Hazri Ab Rashid ${ }^{3}$ and Jung-Hoon Sohn ${ }^{1,2, *}$ \\ 1 Synthetic Biology \& Bioengineering Research Center, Korea Research Institute of Bioscience and \\ Biotechnology (KRIBB), 125 Gwahak-ro, Yuseong-gu, Daejeon 34141, Korea; abedozo@gmail.com (Z.H.I.); \\ hoon@kribb.re.kr (J.-H.B.); werfg@kribb.re.kr (S.-H.L.); bhsung@kribb.re.kr (B.H.S.) \\ 2 Department of Biosystems and Bioengineering, KRIBB School of Biotechnology, Korea University of Science \\ and Technology (UST), 217 Gajeong-ro, Yuseong-gu, Daejeon 34113, Korea \\ 3 Industrial Biotechnology Research Centre, SIRIM Berhad, No.1, Persiaran Dato' Menteri, Section 2, \\ P.O. Box 7035, 40700 Shah Alam, Malaysia; ahazri@sirim.my \\ * Correspondence: sohn4090@kribb.re.kr; Tel.: +82-42-860-4458; Fax: +82-42-879-8499 \\ + These authors contributed equally to this study.
}

Received: 5 March 2020; Accepted: 5 April 2020; Published: 7 April 2020

\begin{abstract}
A lipolytic yeast Candida aaseri SH14 that can utilise long-chain fatty acids as the sole carbon source was isolated from oil palm compost. To develop this strain as a platform yeast for the production of bio-based chemicals from renewable plant oils, a genetic manipulation system using CRISPR-Cas9 was developed. Episomal vectors for expression of Cas9 and sgRNA were constructed using an autonomously replicating sequence isolated from C. aaseri SH14. This system guaranteed temporal expression of Cas9 for genetic manipulation and rapid curing of the vector from transformed strains. A $\beta$-oxidation mutant was directly constructed by simultaneous disruption of six copies of acyl-CoA oxidases genes $(A O X 2, A O X 4$ and $A O X 5)$ in diploid cells using a single sgRNA with $70 \%$ efficiency and the Cas9 vector was efficiently removed. Blocking of $\beta$-oxidation in the triple AOX mutant was confirmed by the accumulation of dodecanedioic acid from dodecane. Targeted integration of the expression cassette for C. aaseri lipase 2 was demonstrated with $60 \%$ efficiency using this CRISPR-Cas9 system. This genome engineering tool could accelerate industrial application of C. aaseri $\mathrm{SH} 14$ for production of bio-based chemicals from renewable oils.
\end{abstract}

Keywords: Candida aaseri; CRISPR; genetic manipulation; acyl-CoA oxidase

\section{Introduction}

Yeasts are unicellular eukaryotes with a long history of use in the biopharmaceutics and food industries [1,2]. Owing to their robust physiology in harsh conditions, such as elevated temperatures and low $\mathrm{pH}$, many different yeasts have also been explored for the production of renewable bio-based chemicals to replace environmentally detrimental petrochemicals [3]. Bio-based production of long-chain dicarboxylic acids for polymer-based industries, including dodecanedioic acid (DDDA, C12), sebacic acid (C10), suberic acid (C8) and adipic acid (C6), has attracted considerable interest as a sustainable alternative to petroleum-based chemicals. Among them, adipic acid (1,6 hexanedioic acid) is an important building block for the manufacture of nylon 6,6, thermoplastic polyurethane resins, plasticisers, adhesives and synthetic lubricants [4]. The estimated global production of adipic acid in 2016 was 3.3 million tons, with an annual growth of $3 \%$ to $3.5 \%$ per year $[5,6]$.

For bio-based production of adipic acid from renewable feedstocks, several methods have been developed in microorganisms. Escherichia coli and Saccharomyces cerevisiae were engineered to produce 
adipic acid from glucose by introduction of the reverse adipate-degradation pathway [7] and cis, cis-muconic acid pathway [8], respectively. Alkanes or fatty acids can be converted to the corresponding dicarboxylic acids by terminal oxidation of the $\omega$-oxidation pathway and then used as carbon source via $\beta$-oxidation pathway in yeast. Different dicarboxylic acids were produced from alkanes or fatty acids by blocking or engineering of the $\beta$-oxidation pathway of a pathogenic yeast Candida tropicalis and the conversion efficiency was much improved by amplification of the $\omega$-oxidation pathway [9]. Therefore, to produce long-chain dicarboxylic acids from renewable plant oils using microbial cell factories, a safe and robust strain that is able to utilise various plant oils as carbon sources and tolerant to organic acids is desirable [10,11].

A lipolytic yeast, Candida aaseri SH14, was previously isolated from the compost of oil palm empty fruit bunches for the production of bio-based chemicals [12]. The type strains of Candida aaseri (syn. Candida butyri) were isolated from butter by Nakase [13]. Until now, there have been few reports about this strain. A few years ago, C. pseudoaaseri closely related to C. aaseri was isolated from a clinical sample of an immunocompromised cancer patient on the basis of the nucleotide divergence [14]. Unlike C. aaseri, which is susceptible to all tested antifungals, C. pseudoaaseri was resistant to flucytosine and distinguished from $C$. aaseri by its colony morphology. Since $C$. aaseri SH14 strain shows outstanding lipolytic activity which derives from lipases (Figure S1), this strain can utilize various fatty acids from plant oils directly and long-chain alkanes as sole carbon sources and is resistant to high concentrations of organic acids (Figure S2). Thus, C. aaseri SH14 could be a microbial factory for the production of dicarboxylic acids, including adipic acid and other renewable chemicals, from plant oils.

However, this potential is limited by the lack of knowledge of the molecular mechanisms of C. aaseri SH14. Recently, we identified eight putative genes for lipase from this diploid strain in the draft genome sequencing and found that this strain belongs to the CTG:Ser group of Candida species [12]. Genetic engineering tools are further required to develop this strain as a platform host for the production of bio-based chemicals. Traditionally, the URA blast and Cre/LoxP systems have been used for multiple gene disruption in yeast $[15,16]$. However, these systems are suitable only for the metabolic engineering of established haploid strains having high homologous recombination activity, such as S. cerevisiae, Schizosaccharomyces pombe and Kluyveromyces lactis. Diploid or polyploid industrial yeasts are rarely suitable because the simultaneous modification of multiple alleles is an extremely inefficient and time-consuming way to obtain a null mutant. The discovery of clustered regulatory interspaced short palindromic repeats (CRISPR) system in bacteria [17] has been followed by its widespread use for numerous applications related to functional genomic studies in various organisms owing to its high efficiency, ease of operation and more rapid operation [18-20]. Several groups of organisms have been genetically edited using the modified type II CRISPR system [21-23]. The system is composed of a CRISPR-associated nuclease (Cas9) and a synthetic guide RNA (sgRNA) that confers target sequence specificity to Cas9 [24].

The CRISPR-Cas9 system has been applied in many different yeasts $[25,26]$. The core challenge of CRISPR-Cas9 for application in nonconventional yeast is how to express Cas9 and sgRNA. Generally, the expression vector for Cas9 and sgRNA is preferentially integrated into the genome if natural plasmid is not available. The first application of CRISPR-Cas9 in nonconventional yeast was in C. albicans [27]. A modified Cas 9 and sgRNA expression cassette was integrated into a specific locus of the genome using a recyclable nourseothricin $\mathrm{N}$-acetyl transferase (NAT1) cassette. After modification of the target genes, flippase was further expressed to remove the genome-integrated NAT1 and Cas9 genes. In this version, the removal of genomic Cas 9 was not efficient enough. A plasmid based CRISPR-Cas9 system was successfully developed in the nonconventional yeast $C$. parapsilosis [28]. Transient expression of Cas9 using a plasmid with an autonomously replicating sequence (ARS) obtained from the genome of C. parapsilosis has enabled the efficient multiple editing of target genes. Unlike these strains, Cas9 expression vectors of $S$. cerevisiae were directly used for genome engineering of $C$. glabrata without modifications [29]. Our initial approach using a plasmid system of S. cerevisiae in C. aaseri SH14 was not successful. There might be many unknown factors determining the efficiency of gene editing using 
CRISPR-Cas9 in nontraditional Candida yeasts due to the broad spectrum of Candida spp. Consequently, it is necessary to develop species-specific CRISPR-Cas9 systems for efficient genome engineering of $C$. aaseri $\mathrm{SH} 14$.

Here, we have developed a CRISPR-Cas9 system for genetic manipulation of C. aaseri SH14. The system consists of Candida-compatible Cas9 gene, ARS, gRNA expression cassette and nourseothricin (NTC) resistance selection marker in a single episomal plasmid. Multiple gene disruption, targeted integration of expression cassette and rapid curing of CRISPR-Cas9 plasmid were successfully demonstrated.

\section{Materials and Methods}

\subsection{Strain, Chemicals and Media}

C. aaseri SH14 and their mutant strains were cultured in YPD (1\% yeast extract, $2 \%$ Bacto peptone and $2 \%$ glucose). Selection of URA+ transformants was performed on a synthetic complete medium lacking uracil (SC-ura; 0.67\% yeast nitrogen base without amino acids, $0.077 \%$ ura dropout supplement, $2 \%$ glucose and $2 \%$ Bacto agar). For antibiotic media, YPD was supplemented with an appropriate concentration of nourseothricin (NTC, Sigma-Aldrich, St. Louis, MO, USA) or hygromycin (Sigma-Aldrich). SC+5-FOA contained SC supplemented with $0.01 \%(w / v)$ uracil (Sigma-Aldrich) and 0.05\% 5-FOA (Duchefa Biochemies, Haarlem, The Netherlands). For SC with methyl laurate (SC+ML), glucose was replaced with $1 \%(v / v)$ methyl laurate (TCI Chemicals, Tokyo, Japan) equilibrated at $\mathrm{pH} 7.0$ with $\mathrm{NaOH}$. Lipase secreting transformants were screened using YP supplemented with $1 \%$ tributyrin (Sigma-Aldrich). Escherichia coli DH5 $\alpha$ [F- lacZ $\Delta$ M15 hsdR17(r-m-) gyrA36] was used for general recombinant DNA techniques. All other chemicals were purchased from Sigma-Aldrich. Restriction enzymes were purchased from New England Biolabs (Ipswich, MA, USA). DNA purification was conducted using the Wizard®SV Gel and PCR Clean-Up Systems (Promega, Madison, WI, USA).

\subsection{Cloning of ARS Element and Construction of Episomal CRISPR-Cas9 Vector}

To obtain genomic ARS elements of C. aaseri SH14, a C. aaseri SH14 genomic library was constructed. The genomic DNA was partially digested with Sau3AI and DNA fragments ranging from $1.5 \mathrm{~kb}$ to 3 $\mathrm{kb}$ were cloned into the BamHI site of pBluescriptII KS (+) containing the NAT1 under the control of the URA3 promoter and terminator. Plasmid digestion analysis showed that $>80 \%$ clones contained average DNA fragments of $1.2 \mathrm{~kb}$ and approximately $3.7 \times 10^{8}$ clones were screened for ARS elements. This library provided a $1.4 \times$ coverage of the $C$. aaseri SH14 genome size. Plasmids were rescued from $C$. aaseri SH14 transformants that survived on the selective medium by back-transformation of E. coli with the total DNA harvested from C. aaseri SH14 transformants. For the construction of the CRIPSR-Cas9 vector (pAN-Cas9), we designed the CRISPR-Cas9 expression system together with sgRNA containing a target-specific sequence. For preparation of the Cas9 gene, the sequence retrieved from p414-TEF1p-Cas9-CYC1t [30] was edited based on the optimised codon of C. aaseri and synthesised with nuclear localization signal (NLS) by Bioneer (Daejeon, Korea). For the constitutive expression of Cas9, Cas9-NLS was flanked with the GAPDH promoter and terminator and cloned into the XbaI and XhoI sites of pBluescript-II KS (+) plasmid containing ARS.

For the construction of the sgRNA expression cassette, a fragment fused with HH ribozyme, gRNA scaffold and HDV ribozyme was synthesised as previously described [31]. TEF1 promoter and the $\mathrm{CYC1}$ terminator were used for transcription of the HH-sgRNA scaffold-HDV. The sgRNA target sequences (20bp) were incorporated into the pAN-Cas9 vector by overlap extension PCR (Figure S3). Sequences of constructed vector were confirmed by Genotech (Daejeon, Korea). All primers and vectors used in this study were listed in Tables S1 and S2. 


\subsection{Transformation and Characterization of Transformants}

The transformation method described by Kondo et al. [31] was applied to C. aaseri SH14 with a slight modification. Cells were grown in YPD medium until the optical density at $600 \mathrm{~nm}\left(\mathrm{OD}_{600}\right)$ reached 1.0 and then were harvested by centrifugation at $3500 \mathrm{rpm}$ for $20 \mathrm{~min}$. Cells were suspended into transformation buffer containing $5 \mathrm{mM}$ lithium acetate, $0.5 \mathrm{M}$ sorbitol, $10 \mathrm{mM}$ Tris- $\mathrm{HCl}(\mathrm{pH} 7.4)$ and $1 \mathrm{mM}$ EDTA, and then $0.01 \mathrm{vol}$ of freshly prepared $1 \mathrm{M}$ dithiothreitol (DTT) was added. After one-hour incubation at room temperature, cells were washed with $1 \mathrm{M}$ sorbitol three times and resuspended in $0.5 \mathrm{~mL}$ of $1 \mathrm{M}$ sorbitol for transformation. For each transformation, $0.5 \mu \mathrm{g}$ of plasmid DNA was mixed with $100 \mu \mathrm{L}$ of competent cells in a $2 \mathrm{~mm}$ electroporation cuvette and incubated on ice for $10 \mathrm{~min}$. Electroporation was performed using the Gene-pulser II (Bio-Rad, Hercules, CA, USA) at $2.25 \mathrm{kV}, 50 \mu \mathrm{F}$ and $200 \Omega$. After electroporation, $1 \mathrm{~mL}$ of YPD containing $1 \mathrm{M}$ sorbitol was added and incubated at $30^{\circ} \mathrm{C}$ overnight. Transformants carrying pAN-Cas9sgRNA plasmid were screened on YPD supplemented with $20 \mu \mathrm{g} / \mathrm{mL}$ NTC.

Positive transformants were confirmed by colony PCR. Randomly selected colonies were suspended in lysis buffer (10 $\mathrm{mM}$ sodium phosphate and $2 \mathrm{mg} / \mathrm{mL}$ lyticase) and incubated at $37^{\circ} \mathrm{C}$ for $30 \mathrm{~min}$. Then, proteinase $\mathrm{K}$ solution $(2 \mathrm{mg} / \mathrm{mL})$ was added to the mixture and incubated at $50{ }^{\circ} \mathrm{C}$ for $10 \mathrm{~min}$ followed by inactivation of the proteinase at $80{ }^{\circ} \mathrm{C}$ for $10 \mathrm{~min}$. Cell lysate $(1 \mu \mathrm{L})$ was used as a template for PCR analysis.

\subsection{Screening of Recombinant C. aaseri SH14 Overexpressing CaLIP2}

C. aaseri SH14 lipase2 (CaLIP2) expression cassette was designed and used in gene knock-in using Cas9 system. CaLIP2 expression cassette contains CaLIP2 gene fused with constitutive TEF1 promoter of $C$. aaseri and CYC1 terminator. For confirmation of additionally expressed CaLIP2, hexa histidine-tag was included at C-terminus of CaLIP2. This cassette was flanked with a 380 bp homology arms of CaAOX2 gene near sgRNA targeting site at both ends. This fragment was used as a donor DNA. To integrate CaLIP2 expression cassette into CaAOX2 locus, pAN-Cas9-gAOX2 plasmid which targeted to a specific $A O X 2$ locus was co-transformed with donor DNA. Integration into the targeted locus was confirmed by PCR analysis using primers located on the cassette (LIP2R) and primers located at the upper site of CaAOX2 locus (AOX2F).

Cells obtained after transformation of pAN-Cas9-gAOX2 plasmid and CaLIP2 cassette were dotted onto YP supplemented with $1 \%$ tributyrin and incubated at $30^{\circ} \mathrm{C}$ to identify lipase secreting transformants based on the development of a clear halo. Lipase expressing transformants were cultured in $\mathrm{YP}$ broth for $40 \mathrm{~h}$ and culture broth was used to determine lipase activity, which was determined using $p$-nitrophenyl palmitate ( $p$-NPP) as substrate [32]. The reaction mixture (50 $\mathrm{mM}$ Tris- $\mathrm{HCl}$ buffer, $\mathrm{pH}$ 8.0, 2 mM $p$-NPP and culture broth) was incubated at room temperature for 5 min and $p$-nitrophenol released by lipase was quantified using a spectrophotometer according to the absorbance at $405 \mathrm{~nm}$. One unit of lipase activity was defined as the amount of enzyme needed to release $1 \mu \mathrm{mol}$ of $p$-nitrophenol per minute. Transformants were cultivated in a $10 \mathrm{~mL}$ test tube containing $2 \mathrm{~mL}$ YPD broth medium for $40 \mathrm{~h}$. Then, $0.6 \mathrm{~mL}$ of culture supernatant was precipitated with $0.4 \mathrm{~mL}$ of cold acetone and analysed by electrophoresis on $12 \%$ polyacrylamide gels under denaturing conditions by staining with Coomassie blue. The western blot analysis was performed using anti-His (Sigma Aldrich) after dilution of 1/1000 times. Protein samples were electrophoresed and transferred to nitrocellulose membrane using iBlotß2 Dry Blotting System (Thermo Fisher Scientific) following manufacturer's instructions. The reacting antibodies were detected with anti-mouse immunoglobulins conjugated to alkaline phosphatase (Sigma Aldrich).

\subsection{Analysis of dodecanedioic Acid (DDDA)}

Analysis of DDDA was performed using the method of Funk et al [33] with slight modifications. The culture broth was adjusted to $\mathrm{pH} 10$ with sodium hydroxide and boiled for 5 min. Supernatant was 
acidified with 2N sulfuric acid and DDDA was extracted with 2 volume of MTBE (methly tert-butyl ether) for $2 \mathrm{~h}$. Next step, extracted DDDA was mixed with 0.25 volume of trimethylsilyl trifluoroacetamide and silylated at $90^{\circ} \mathrm{C}$ for $50 \mathrm{~min}$. DDDA and dodecane were measured by gas chromatography using HP5 column and Agilent 7890A gas chromatograph system (Agilent, Technologies, Yarnton, England) equipped with a flame ionization detector (FID) and a HP5 capillary column (30 m length, $0.32 \mathrm{~mm}$ ID, $0.25 \mu \mathrm{m}$ film thickness). The He carrier gas flow was set to $3 \mathrm{~mL} / \mathrm{min}$ and increased from $70{ }^{\circ} \mathrm{C}$ to $185^{\circ} \mathrm{C}$ at $15^{\circ} \mathrm{C} / \mathrm{min}$, then ramped to $300^{\circ} \mathrm{C}$ at $30^{\circ} \mathrm{C} / \mathrm{min}$ and finally held at $300^{\circ} \mathrm{C}$ for $2 \mathrm{~min}$.

\section{Results}

\subsection{Development of Transformation System for C. aaseri SH14}

To develop a transformation system in C. aaseri, sensitivity to various antibiotics that are frequently used in transformation of yeast were analysed. Two antibiotics, NTC and hygromycin, which inhibited the growth of $C$. aaseri $\mathrm{SH} 14$, were selected and the minimum inhibitory concentrations were determined (Figure S4). Since C. aaseri SH14 showed higher sensitivity to NTC $(10 \mu \mathrm{g} / \mathrm{mL})$ than hygromycin $(100 \mu \mathrm{g} / \mathrm{mL})$, NAT1 gene from Streptomyces noursei was employed as a selection marker gene. In addition, because the NAT1 gene does not contain the CTG codon, which is predominantly translated as serine instead of leucine in CTG:Ser clade species, the gene was expressed under the control of C. aaseri URA3 promoter without modification. To construct an episomal vector of $C$. aaseri $\mathrm{SH} 14$, ARS were isolated from a genomic library of $C$. aaseri SH14 constructed in the pBluescript II KS+ plasmid containing NAT1 gene. C. aaseri SH14 was transformed with the genomic library without linearization and transformants were selected on YPD plates containing $20 \mu \mathrm{g} / \mathrm{mL}$ NTC. After screening of approximately $4 \times 10^{4}$ transformants, two colonies were isolated and plasmids were retrieved from the transformants. Two different ARS elements were identified by DNA sequencing and designated ARS1 (1265 bp) and ARS2 (789 bp) (Figure S5).

To define the core regions of ARS1 and ARS2 responsible for the replication of episomal plasmid, several plasmids containing each deleted ARS fragment were constructed, and their transformation efficiency was checked. Only ARS1-1 (460 bp) and ARS2-4 (324 bp) showed a comparable transformation efficiency with the full-length ARS elements (Figure 1A). The stability of each plasmid containing ARS1, ARS1-1, ARS2 and ARS2-4 was analysed in YPD medium without selection pressure. All tested plasmids completely disappeared after several generations under nonselective condition (Figure 1B). These results implied that the cloned ARSs are useful for the highly efficient transformation and simple elimination of plasmid containing Cas9 expression cassette in C. aaseri SH14. Considering the size of plasmid, the plasmid pAN-ARS2-4 containing ARS2-4 was selected for expression of Cas9.

\subsection{Development of CRISPR-Cas9 Expression System for C. aaseri SH14}

For the development of an easily modifiable CRISPR-Cas9 system in C. aaseri SH14, a single vector system was designed for the expression of Cas9 and sgRNA. Considering the CTG:Ser clade of C. aaseri, a gene coding for a Candida codon optimised Cas9 (Figure S6, 84 CTG changed to CTT or TTA) fused with SV40 nuclear localization signal was synthesised and expressed under the control of the glyceraldehyde 3-phosphate dehydrogenase $(G A P D H)$ promoter of $C$. aaseri SH14. For the expression of sgRNA, RNAP III promoters that do not modify the $5^{\prime}$ and $3^{\prime}$ end of RNA are typically required for correct formation of functional sgRNA. Since the confirmed RNAP III promoters are not available in C. aaseri SH14, the translational elongation factor1 (TEF1) promoter and two different self-cleaving ribozyme regions, hammerhead ribozyme (HH) and hepatitis delta virus (HDV), were used to make a mature sgRNA without post-transcriptional modifications [32]. The Cas9 and sgRNA cassettes were cloned into the pAN-ARS2-4 vector to construct a pAN-Cas9gRNA plasmid (Figure 2A). 
A

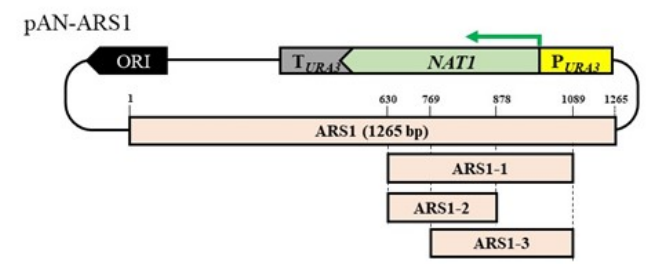

\begin{tabular}{|c|c|}
\hline ARS & $\begin{array}{c}\text { Transformation } \\
\text { efficiency } \\
\text { (cfu/mg DNA) }\end{array}$ \\
\hline ARS1 & $1.36 \times 10^{4} \pm 30$ \\
\hline ARS1-1 & $1.34 \times 10^{4} \pm 34$ \\
\hline ARS1-2 & No colony \\
\hline ARS1-3 & $3.11 \times 10^{3} \pm 41$ \\
\hline
\end{tabular}

pAN-ARS2

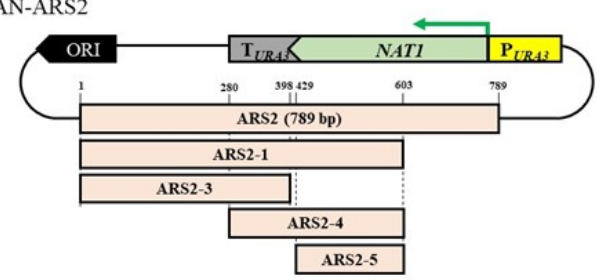

\begin{tabular}{|c|c|}
\hline ARS & $\begin{array}{c}\text { Transformation } \\
\text { efficiency } \\
\text { (cfu/mg DNA) }\end{array}$ \\
\hline ARS2 & $1.36 \times 10^{4} \pm 79$ \\
\hline ARS2-1 & $1.16 \times 10^{4} \pm 58$ \\
\hline ARS2-3 & No colony \\
\hline ARS2-4 & $1.32 \times 10^{4} \pm 35$ \\
\hline ARS2-5 & No colony \\
\hline
\end{tabular}

B

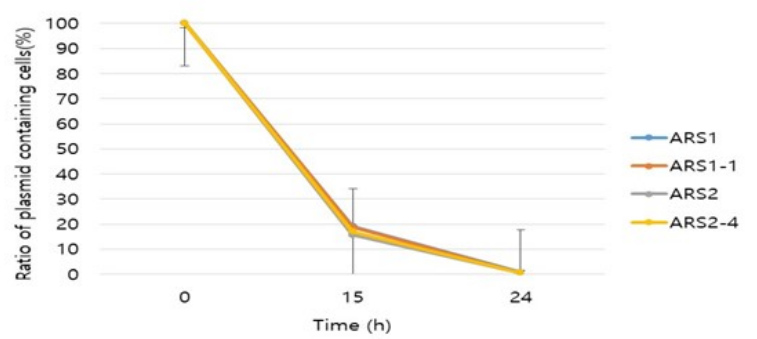

Figure 1. Determination of core domain of ARS1 and ARS2 and stability test of autonomously replicating sequence (ARS) plasmids. (A) Schematic diagram of ARS fragments and transformation efficiency. (B) Stability of different ARS plasmids. Mean values and standard deviations of triplicates are shown.

A

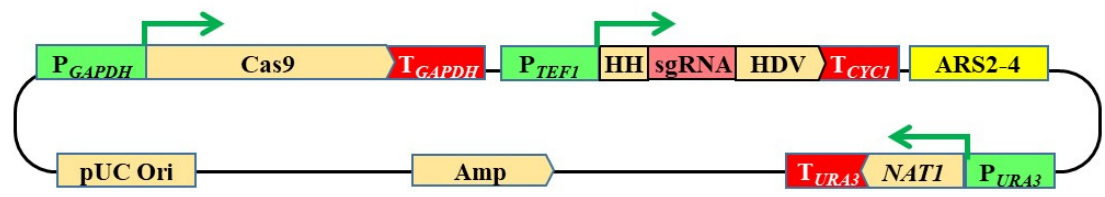

B

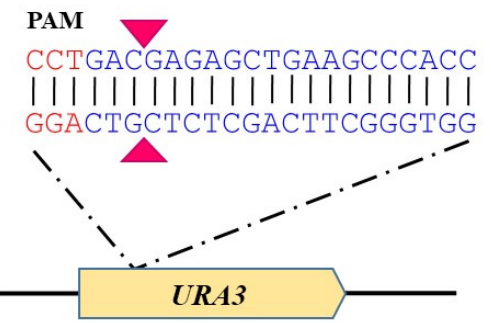

C
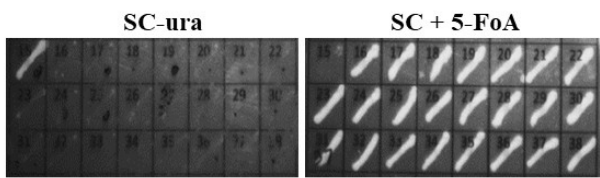

PAM Cut site

D cttcaat tcat actgac gagagct gaagcccacccctctccagtc

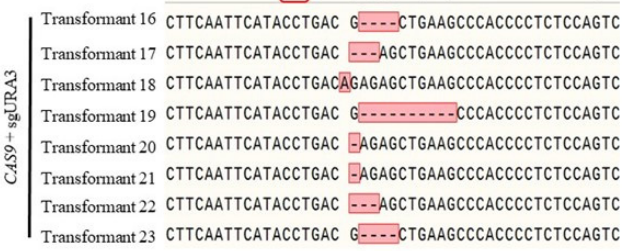

Figure 2. Targeted disruption of CaURA3 using CRISPR-Cas9 system. (A) Schematic diagram of pAN-Cas9gRNA vector. HH; hammerhead ribozyme, HDV; and hepatitis delta virus ribozyme. (B) CaURA3 target sequence generated by ATUM program. Arrowheads indicate Cas9 cleavage site. (C) Conformation of ura3 mutant phenotype on SC-ura and SC+5-FOA plates. (D) Sequence analysis of CaURA3 from eight transformants. Insertions or deletions were indicated (red box).

As a proof of our system, the CaURA3 gene was selected for targeted disruption. Synthesis of the CaURA3 targeting sequence for sgRNA (gURA3) was designed using the ATUM CRISPR gRNA design tool (www.atum.bio/eCommerce/cas9/input) (Figure 2B). To eliminate off-target activity, 
the gRNA sequence generated from ATUM was confirmed by a similarity search against the C. aaseri genome database. C. aaseri SH14 was transformed using pAN-Cas9gURA3 without linearization and transformants were selected on YPD containing $20 \mu \mathrm{g} / \mathrm{mL}$ NTC. Twenty-four randomly selected transformants were transferred to both SC-ura and SC+5-fluoroorotic acid (FOA) plates. All except one of the transformants displayed the uracil auxotrophic phenotype growing on SC+5-FOA medium (Figure 2C). The sequences of CaURA3 from eight transformants recovered from the SC+5-FOA plate were compared with the wild type sequence (Figure 2D). Seven transformants showed 1 to 10 base deletions and one contained one base insertion at the Cas9 cleavage site. Considering C. aaseri SH14 is diploid, two copies of CaURA3 gene were disrupted with nearly 100\% efficiency.

\subsection{Deletion of Multiple Genes by a Single Guide RNA}

In yeast, most of the fatty acid molecules are catabolized to the acetyl-CoA by $\beta$-oxidation pathway or are converted to dicarboxylic acids by $\omega$-oxidation when $\beta$-oxidation is defective [33-36]. Therefore, to produce long-chain dicarboxylic acids from fatty acids, the $\beta$-oxidation pathway should be inactivated to block further oxidation to acetyl-CoA. $\beta$-oxidation is a cyclic degradation of a fatty acid catalysed by acyl-CoA oxidases (AOXs; EC: 1.3.3.6). Three AOX homologues, CaAOX2, CaAOX4 and CaAOX5, were identified from the $C$. aaseri genome by BLAST analysis using known AOX genes. To find conserved regions that could be used as a common target for sgRNA, the nucleotide sequences of the three genes were aligned and a region having exactly the same $20 \mathrm{bp}$ with different protospacer adjacent motif (PAM) sequences was selected (Figure 3A). The sgRNA target sequence of pAN-Cas9gRNA was replaced with this sequence to construct pAN-Cas9gAOX. C. aaseri SH14 was transformed using the plasmid and transformants were selected on YPD containing $20 \mu \mathrm{g} / \mathrm{mL}$ NTC. Since the mutants defective in $\beta$-oxidation were unable to utilize fatty acid as a sole carbon source, transformants were selected by replica plate analysis using SC+Glc and SC+ML containing $1 \%$ glucose and methyl laurate, respectively (Figure 3B). Twenty two of 32 transformants (70\%) were unable to grow on SC+ML. To identify the sequence of AOX genes, specific primers located on each gene were used to amplify a region containing the sgRNA target site. Six randomly selected transformants per AOX gene showing a mutagenic phenotype were analysed. All tested AOX genes revealed indels at specific sgRNA targets (Figure 3C), suggesting that complete disruption of multiple genes of the six copies could be done with an efficiency of approximately $70 \%$ by a single transformation.

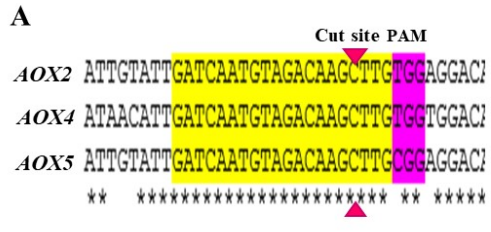

B

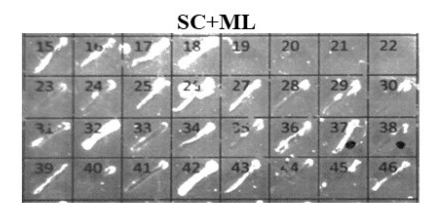

SC+Glc

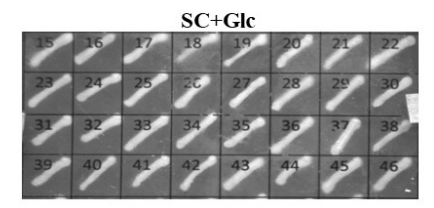

C Cutsite PAM

C CaAOX2 gcagctgattgtattgatcaatgtagacaagc ttgeggagacatggatactctgctt Transformant 23 GCAGCTGATtGTATTGATCAATGTAGACAagCGITGCGGAGGACATGGaTACTCTGCTT Transformant 24 GCAGCTGATTGTATTGATCAATGTAGACAAGCCT TGCGGAGGACATGGATACTCTGCTT Transformant 40 GCAGCTGATtGTATTGATCAATG TAGACAA- - TtGCGGaGGACATGGATACTCTGCTT Transformant 41 GCAGCTGATTGTATTGATCAATGTAGACAAGCG TGGGGAGGACATGGATACTCTGCTT Transformant 44 GCAGCTGATTGTATTGATCAATGTAGACAAGCC] TGCGGAGGACATGGATACTCTGCTT

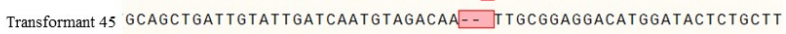
Cut site PAM

CaAOX4 gctgctgattgtattgatcaatgtagacaagc t tg tggaggacatggttattctgct Transformant 23 GCTGCTGATTGTATTGATCAATGTAGACAAGCGTTGTGGAGGACATGGTTATTCTGCTT

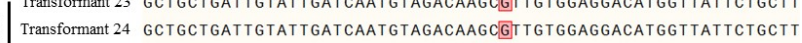

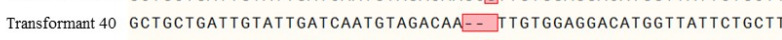

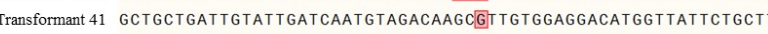
Transformant 44 GCTGCTGATTGTATTGATCAATGTAGACAAGCGT TGTGGAGGACATGGTTATTCTGCT Transformant 45 GCTGCTGATTGTATTGATCAATGTAGACAA - - TTGTGGAGGACATGGTTATTCTGCTT Cut site PAM

CaAOXs act ttaaataacat tgatcaatgtagacaagc ${ }^{t}$ tg gggtggacatggatat tcagct t Transformant 23 ACTTTAAATAACATTGATCAATGTAGACAAGCGT TGTGGTGGACATGGATATTCAGCTT

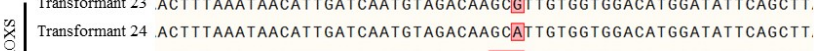

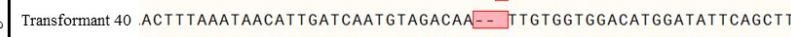
Transformant 41 .ACTTTAAATAACATTGATCAATGTAGACAAGCGITGTGGTGGACATGGATATTCAGCT Transformant 44 . ACTTTAAATAACATTGATCAATG TAGACAAGCATT TGTGGTGGACATGGATATTCAGCTT Transformant 44 . ACTTTAAATAACAT TGATCAATG TAGACAAGCAT TGTGGTGGACATGGATAT TCAGC TT
Transformant 45 .ACTTTAAATAACATTGATCAATGTAGACAA- - TTGTGGTGGACATGGATATCAGC TT

Figure 3. Multiple gene disruption by single gRNA. (A) Sequence alignment of CaAOX2, CaAOX4 and CaAOX5 genes. The conserved gRNA target sequence (20 bp) and PAM sequence were indicated. (B) Growth of mutants on methyl laurate $(\mathrm{SC}+\mathrm{ML})$ and glucose $(\mathrm{SC}+\mathrm{Glc})$ as a sole carbon source.

(C) Sequence analysis of six different mutants. Insertions or deletions were indicated (red box). 
To confirm the induction of the $\omega$-oxidation pathway in a triple aox mutant, conversion of dodecane to dodecanedioic acid (DDDA) was conducted using wild type and triple aox mutant (Figure 4). Contrary to the wild type, DDDA was found in the culture broth of the triple aox mutant, indicating complete inactivation of $\beta$-oxidation. Therefore, six copies of AOX genes in diploid were disrupted with $70 \%$ efficiency using a single sgRNA.

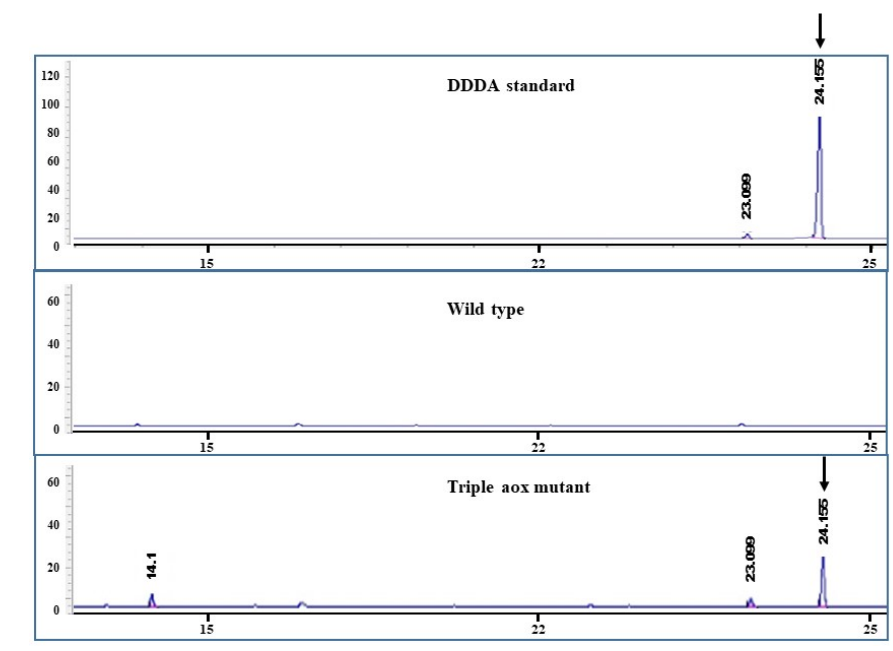

Figure 4. Gas Chromatography analysis for the detection of dodecanedioic acid (DDDA) from dodecane. DDDA peak was detected at 24.15 min from culture broth of triple aox mutant.

\subsection{Cas9 Mediated Targeted Integration and Curing of $p A N-C a s 9 g R N A$ Vector}

Disruption of target genes using non-homologous end joining (NHEJ) and integration of recombinant genes into a specific locus by homologous recombination is an important tool for genetic engineering of industrial yeast. To confirm gene knock-in using the Cas 9 system in C. aaseri SH14, the $C$. aaseri lipase2 (CaLIP2) expression cassette was integrated into the CaAOX2 locus. The C. aaseri $\beta$-oxidation mutant was cotransformed with the Cas 9 vector containing sgRNA expression cassette targeted to CaAOX2 (pAN-Cas9gAOX2) and CaLIP2 expression cassette containing 380 bp homology arms at both ends (Figure 5A, Figure S7). Overexpression of CaLIP2 was distinguished by comparison of the halo zone generated by the wild type and transformants on tributyrin containing medium. Integration of the CaLIP2 expression cassette into the CaAOX2 locus was confirmed by PCR using primers located on the cassette (LIP2R) and primers located at the upper site of CaAOX2 locus (AOX2F). Among the ten transformants showing expanded halo zones, six (60\%) involved cassette integration via homologous recombination. Lipase activity of the positive transformants was over 10-fold higher than that of wild type (Figure 5B). After cultivation of a transformant showing the highest lipase activity, the culture broth was examined by western blot analysis (Figure 5C). Since CaLIP2 contains three putative glycosylation sites, a smear of protein bands larger than $62 \mathrm{kDa}$ was detected in transformant CaL-5. After treatment with deglycosydase (Endo-H), mature CaLIP2 was detected as a $51.3 \mathrm{kDa}$ band. Several protein bands smaller than mature CaLIP2 after deglycosylation might have resulted from proteolysis during cultivation.

For the repetitive use of the Cas9 to modify another target, easy removal of Cas9 and gRNA expression cassettes in the previously engineered cells is indispensable. To remove the pAN-Cas9gAOX2 plasmid from the recombinant strain, the strain was cultivated in YPD medium without NTC for $24 \mathrm{~h}$ and then an equal number of cells were plated on YPD and YPD+20 $\mu \mathrm{g} / \mathrm{mL}$ NTC medium. As shown in Figure 6, no colonies developed on YPD+NTC medium, indicating the complete loss of the plasmid. Consequently, the Cas9 vector developed in this study was useful for the temporal expression of Cas9 nuclease sufficient for a genome editing and also for easy removal of Cas9 vector from the engineered cells. 


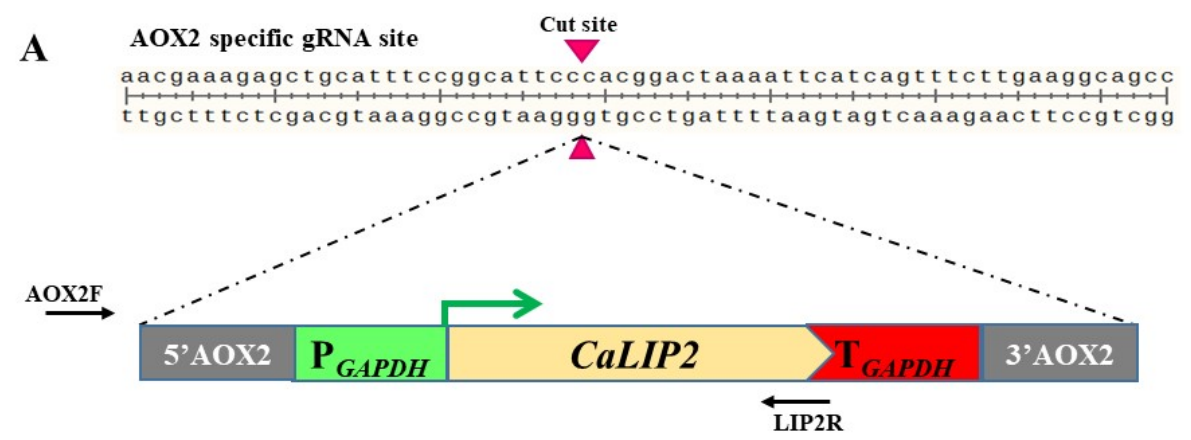

B

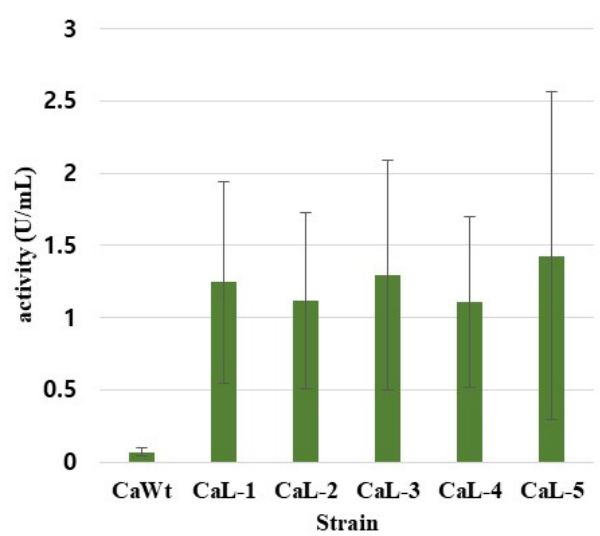

C

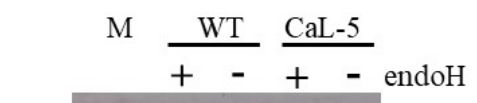

Figure 5. Targeted integration into specific locus by CRISPR-Cas9. (A) Schematic diagram of sgRNA target site and insertion of CaLIP2 cassette via homologous recombination. (B) Lipase activity of selected transformants. (C) Confirmation of CaLIP2 by western blot analysis.

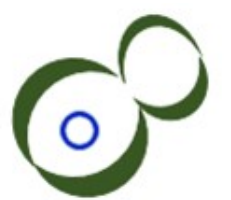

$100 \%$ cells carrying plasmid

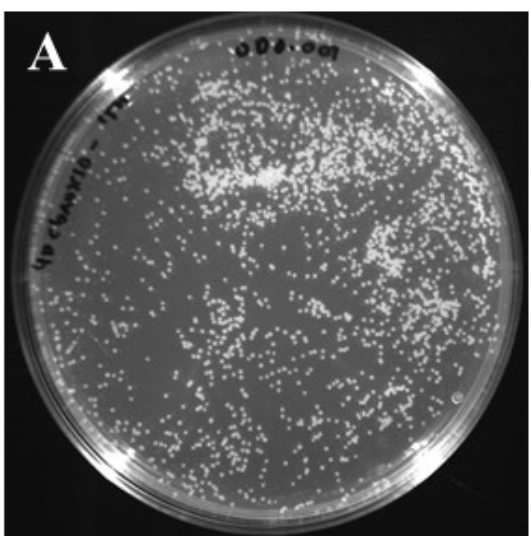

24 hours under non-selective condition

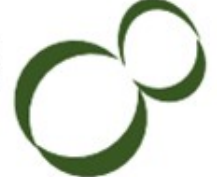

$\sim 99 \%$ plasmid loss

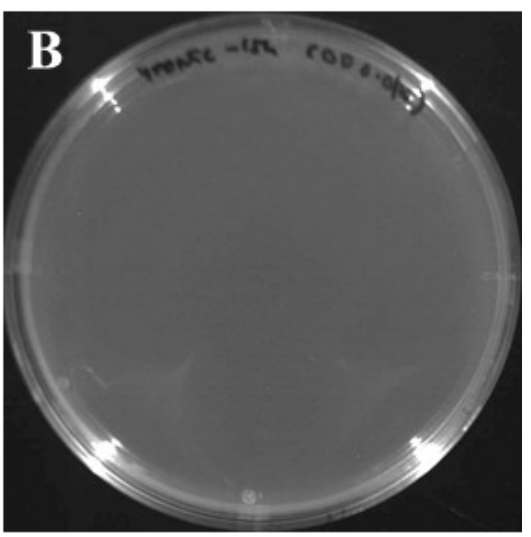

Figure 6. Complete loss of CRISPR-Cas9 plasmid from transformed cells under nonselective condition. Transformed cells harbouring pAN-Cas9gAOX2 vector was cultured in YPD broth without nourseothricin (NTC) for $24 \mathrm{~h}$ and an equal amount of cells was plated on YPD (A) and YPD+20 $\mu \mathrm{g} / \mathrm{mL}$ NTC (B) plate. 


\section{Discussion}

The success of the CRISPR-Cas9 system for genome editing of S. cerevisiae led to many studies of the use of the system for genetic manipulation of various industrial or non-conventional yeasts [37-40]. Especially, Candida spp has attracted considerable interest because it contains several strains showing a significant potential for biotechnological use [41] and clinically important pathogen [42]. Since the members of Candida, such as C. albicans, C. glabrata and C. parapsilosis, are clinically relevant pathogenic yeasts, the CRISPR-Cas9 system was developed to study virulence mechanisms [27-29]. Unlike these strains, C. aaseri SH14 is an environmental species isolated from the compost of oil palm empty fruit bunches. It utilises various fatty acids and alkanes as sole carbon sources. Therefore, this strain could potentially be a platform host for the microbial production of chemicals from renewable oils.

We developed a CRISPR-Cas9 system for the efficient genome engineering of C. aaseri SH14. The system consists of a Candida-compatible Cas9 gene, sgRNA expression cassette, NAT1 marker and ARS in an episomal vector. For easy elimination of the Cas9 and sgRNA cassettes after editing a target locus, both were incorporated in a single episomal vector rather than being integrated into the genome. Because there is no natural plasmid in C. aaseri SH14, we developed an artificial plasmid using ARS elements isolated from a genomic DNA library. Two ARS elements, ARS1 and ARS2, were isolated and their ARS core regions were determined. The core regions of ARS1 and ARS2 were enriched in A-T $(>70 \%)$, which is a typical feature of ARS required for the initiation of replication. Artificial plasmids harbouring both ARSs greatly improved the transformation efficiency in C. aaseri SH14 but were stably maintained only under the selective condition.

Functional expressions of Cas9 and sgRNA are necessary for this system to work. Since C. aaseri SH14 is a CTG:Ser clade, all 84 CTG codons found in the Cas9 gene were changed to CTT or TTA and were expressed under the control of a strong and constitutive GAPDH promoter. As found in other strains, expression of Cas9 protein was also detrimental to C. aaseri SH14 and severely affected growth (Figure S8). The negative effect of Cas9 on cell growth could be eliminated by the easy curing of the Cas9 plasmid from C. aaseri SH14. To express sgRNA without post-transcriptional modifications, RNA polymerase II promoter was used with a self-cleavage sequence, such as HH and HDV ribozymes.

As a proof of concept for multiple gene disruption using this system, disruption of three different AOX genes (six copies in diploids) was done using a single sgRNA. Since three AOX genes were found in C. aaseri SH14 for fatty acid assimilation through $\beta$-oxidation and they are highly conserved, a common target for sgRNA synthesis was selected to make the $\beta$-oxidation mutant by a single transformation of the sgRNA vector. All AOX genes of the selected transformants were abolished with indels. Selected strains showed a typical phenotype of $\beta$-oxidation mutants, namely the inability to grow on a fatty acid, such as methyl laurate or oleic acid, supplied as the sole carbon source. When dodecane was supplied as a carbon source, DDDA was detected only in the triple aox mutant implying inactivation of $\beta$-oxidation pathway. However, the conversion yield was very low compared to that of C. tropicalis [9]. In case of C. tropicalis, the $\omega$-oxidation pathway was amplified by overexpression of the cytochrome $\mathrm{P} 450$ monooxygenase and NADPH-cytochrome reductase genes encoding the rate-limiting steps of the $\omega$-oxidation pathway. Therefore, further engineering of the $\omega$-oxidation pathway of $C$. aaseri SH14 triple aox mutant might be required to improve the conversion yield. From the genome sequence analysis, four genes with high similarity to cytochrome P450 monooxygenase were identified. Engineering of the $\omega$-oxidation pathway $C$. aaseri SH14 using these candidates is underway.

Considering that $C$. aaseri SH14 is diploid, six copies of AOX genes were disrupted with $70 \%$ efficiency using a single sgRNA. Since $\beta$-oxidation is catalysed by several acyl-CoA oxidase isozymes, several AOX genes were identified in plants, animals and microorganisms. Especially, in the yeast Yarrowia lipolytica, five AOX genes have been identified [43]. Besides, designing of common sgRNA is possible because the nucleotide sequence of these genes is highly conserved. Therefore, the approach used in this study would be useful for the construction of $\beta$-oxidation mutant in various strains.

In the beginning of this study, we tried to make a uracil auxotrophic mutant by a traditional method of homologous recombination using a single disruption cassette consisting of the NAT1 gene 
flanked by $1 \mathrm{~kb}$ homologous regions. Although many transformants (more than 160 colonies) were obtained by NTC selection, it was extremely hard to find a correct integrant of the NAT1 gene into the CaURA3 locus with only 13 correct transformants from 120 tested colonies. The finding implies that homologous recombination was overwhelmed by non-homologous recombination in C. aaseri SH14. Therefore, targeted integration via homologous recombination in C. aaseri SH14 was a problem that needed to be solved. Since CRISPR-Cas9-induced DSB has accelerated homologous recombination in many kinds of yeast [29,38,40], we demonstrated gene knock-in by homologous recombination at specific site of $A O X 2$ locus by CRISPR-Cas9. The CaLIP2 cassette without selection marker surrounded with 380 bp of CaAOX2 5' and 3' flanking regions was co-transformed with the Cas9 vector containing the sgRNA expression cassette targeted to CaAOX2. Although a number of ectopic integrations of CaLIP2 cassette by non-homologous recombination (40\%) occurred, the correct integration by homologous recombination $(60 \%)$ surpassed non-homologous recombination. This efficiency could guarantee genetic manipulation of $\mathrm{C}$. aaseri $\mathrm{SH} 14$ by homologous recombination.

In mammalian CRISPR-Cas9 systems, off-target effects that related to the lifetime of Cas9 in a cell has been a major problem awaiting a solution $[44,45]$ Although we did not confirm off-target editing in C. aaseri SH14, this side effect of CRISPR-Cas9 systems could be minimised in our system because of the instability of ARS, facilitating rapid curing of the Cas9 vector.

In conclusion, we have developed a single plasmid based CRISPR-Cas9 system for efficient genetic manipulation of $C$. aaseri SH14. The system could accelerate the development of various engineered strains capable of producing valuable bio-based chemicals from renewable oils.

Supplementary Materials: Supplementary materials can be found at http://www.mdpi.com/2076-2607/8/4/526/s1. Table S1: Oligonucleotide used for sequencing and amplification of sgRNAs, Table S2: Plasmid used in this study. Figure S1: Lipase activity of 16 yeasts isolated from compost of empty fruit bunches of palm oil, Figure S2: Utilization of long-chain fatty acids and alkanes by different yeasts and resistance to organic acids, Figure S3: Construction of sgRNA expression vector, Figure S4: Antibiotic sensitivity test of C. aaseri SH14, Figure S5: Sequences of ARS1 and ARS2, Figure S6: Sequence of C. aaseri codon optimised Cas9 gene, Figure S7: Integration of CaLIP2 overexpression cassette at sgAOX2 via NHEJ, and Figure S8: Effect of Cas9 expression on the growth of C. aaseri SH14.

Author Contributions: Z.H.I. and J.-H.B. performed the data curation, formal analysis, investigation, methodology and writing-original draft preparation. S.-H.L. and B.H.S. performed investigation and methodology. J.-H.S. and A.H.A.R. provided conceptualization and supervision, J.-H.S. and B.H.S. provided funding acquisition, project administration, resources, writing-review and editing. All authors have read and agreed to the published version of the manuscript.

Funding: This work was supported by the Intelligent Synthetic Biology Center of the Global Frontier Program (2015M3A6A8065831), the Basic Science Research Program (2019R1A2C1090726) through the National Research Foundation, the Cooperative Research Program for Agriculture Science and Technology Development (PJ0149382020) through the Rural Development Administration of Korea and the Research Initiative Program of KRIBB.

Conflicts of Interest: The authors declare no conflict of interest.

\section{References}

1. Kim, H.; Yoo, S.J.; Kang, H.A. Yeast synthetic biology for the production of recombinant therapeutic proteins. FEMS Yeast Res. 2015, 15, 1-16. [CrossRef]

2. Darvishi, F.; Fathi, Z.; Ariana, M.; Moradi, H. Yarrowia lipolytica as a workhorse for biofuel production. Biochem. Eng. J. 2017, 127, 87-96. [CrossRef]

3. Nielsen, J.; Larsson, C.; van Maris, A.; Pronk, J. Metabolic engineering of yeast for production of fuels and chemicals. Curr. Opin. Biotechnol. 2013, 24, 398-404. [CrossRef]

4. Deng, Y.; Ma, L.; Mao, Y. Biological production of adipic acid from renewable substrates: Current and future methods. Biochem. Eng. J. 2016, 105, 16-26. [CrossRef]

5. Bart, J.C.J.; Cavallaro, S. Transiting from Adipic acid to bioadipic acid. 1, petroleum-based processes. Ind. Eng. Chem. Res. 2015, 54, 1-46. [CrossRef]

6. Polen, T.; Spelberg, M.; Bott, M. Toward biotechnological production of adipic acid and precursors from biorenewables. J. Biotechnol. 2013, 167, 75-84. [CrossRef] 
7. Zhao, M.; Huang, D.; Zhang, X.; Koffas, M.A.; Zhou, J.; Deng, Y. Metabolic engineering of Escherichia coli for producing adipic acid through the reverse adipate-degradation pathway. Metab. Eng. 2018, 47, $254-262$. [CrossRef]

8. Sun, J.; Raza, M.; Sun, X.; Yuan, Q. Biosynthesis of adipic acid via microaerobic hydrogenation of cis,cis-muconic acid by oxygen-sensitive enoate reductase. J. Biotechnol. 2018, 280, 49-54. [CrossRef]

9. Picataggio, S.; Rohrer, T.; Deanda, K.; Lanning, D.; Reynolds, R.; Mielenz, J.; Eirich, L.D. Metabolic engineering of Candida tropicalis for the production of long-chain dicarboxylic acids. Nat. Biotechnol. 1992, 10, 894-898. [CrossRef]

10. Karlsson, E.; Mapelli, V.; Olsson, L. Adipic acid tolerance screening for potential adipic acid production hosts. Microb. Cell Fact. 2017, 16, 20. [CrossRef]

11. Tsuge, Y.; Kawaguchi, H.; Sasaki, K.; Kondo, A. Engineering cell factories for producing building block chemicals for bio-polymer synthesis. Microb. Cell Fact. 2016, 15, 19. [CrossRef] [PubMed]

12. Lee, S.H.; Jeong, H.; Ko, H.J.; Bae, J.H.; Ibrahim, Z.H.; Sung, B.H.; Sohn, J.H. Draft Genome Sequence of a Lipolytic Yeast. Candida aaseri SH-14. Genome Announc. 2018, 5, e00373-17. [CrossRef] [PubMed]

13. Nakase, T. Four new yeast found in Japan. J. Gen. Appl. Microbiol. 1971, 17, 469-478. [CrossRef]

14. Pfüller, R.; Gräser, Y.; Erhard, M.; Groenewald, M. A novel flucytosine-resistant yeast species, Candida pseudoaaseri, causes disease in a cancer patient. J. Clin. Microbiol. 2011, 49, 4195-4202. [CrossRef] [PubMed]

15. Ribeiro, O.; Gombert, A.K.; Teixeira, J.A.; Domingues, L. Application of the Cre-loxP system for multiple gene disruption in the yeast Kluyveromyces marxianus. J. Biotechnol. 2007, 131, 20-26. [CrossRef]

16. Staab, J.F.; Sundstrom, P. URA3 as a selectable marker for disruption and virulence assessment of Candida albicans genes. Trends Microbiol. 2003, 11, 69-73. [CrossRef]

17. Jinek, M.; Chylinski, K.; Fonfara, I.; Hauer, M.; Doudna, J.A.; Charpentier, E. A Programmable Dual-RNA-Guided DNA Endonuclease in Adaptive Bacterial Immunity. Science 2012, 337, 816-822. [CrossRef]

18. Hsu, P.D.; Lander, E.S.; Zhang, F. Development and applications of CRISPR-Cas9 for genome engineering. Cell 2014, 157, 1262-1278. [CrossRef]

19. Stovicek, V.; Borodina, I.; Forster, J. CRISPR-Cas system enables fast and simple genome editing of industrial Saccharomyces cerevisiae strains. Metab. Eng. Commun. 2015, 2, 13-22. [CrossRef]

20. Jakočiunas, T.; Jensen, M.K.; Keasling, J.D. CRISPR/Cas9 advances engineering of microbial cell factories. Metab. Eng. 2016, 34, 44-59. [CrossRef]

21. Xie, K.; Yang, Y. RNA-Guided genome editing in plants using a CRISPR-Cas system. Mol. Plant 2013, 6, 1975-1983. [CrossRef]

22. Rahdar, M.; McMahon, M.A.; Prakash, T.P.; Swayze, E.E.; Bennett, C.F.; Cleveland, D.W. Synthetic CRISPR RNA-Cas9-guided genome editing in human cells. Proc. Natl. Acad. Sci. USA 2015, 112, E7110-E7117. [CrossRef]

23. Wang, L.; Deng, A.; Zhang, Y.; Liu, S.; Liang, Y.; Bai, H.; Cui, D.; Qiu, Q.; Shang, X.; Yang, Z.; et al. Efficient CRISPR-Cas9 mediated multiplex genome editing in yeasts. Biotechnol. Biofuels 2018, 11, 1-16. [CrossRef]

24. Jiang, F.; Doudna, J.A. CRISPR-Cas9 Structures and Mechanisms. Annu. Rev. Biophys. 2017, 505-531. [CrossRef]

25. Weninger, A.; Hatzl, A.M.; Schmid, C.; Vogl, T.; Glieder, A. Combinatorial optimization of CRISPR/Cas9 expression enables precision genome engineering in the methylotrophic yeast Pichia pastoris. J. Biotechnol. 2016, 235, 139-149. [CrossRef]

26. Horwitz, A.A.; Walter, J.M.; Schubert, M.G.; Kung, S.H.; Hawkins, K.; Platt, D.M.; Hernday, A.D.; Mahatdejkul-Meadows, T.; Szeto, W.; Chandran, S.S.; et al. Efficient Multiplexed Integration of Synergistic Alleles and Metabolic Pathways in Yeasts via CRISPR-Cas. Cell Syst. 2015, 1, 88-96. [CrossRef]

27. Vyas, V.K.; Barrasa, M.I.; Fink, G.R. A Candida albicans CRISPR system permits genetic engineering of essential genes and gene families. Sci. Adv. 2015, 1, e1500248. [CrossRef]

28. Lombardi, L.; Turner, S.A.; Zhao, F.; Butler, G. Gene editing in clinical isolates of Candida parapsilosis using CRISPR/Cas9. Sci. Rep. 2017, 7, 1-11. [CrossRef]

29. Enkler, L.; Richer, D.; Marchand, A.L.; Ferrandon, D.; Jossinet, F. Genome engineering in the yeast pathogen Candida glabrata using the CRISPR-Cas9 system. Sci. Rep. 2016, 6, 35766. [CrossRef]

30. DiCarlo, J.E.; Norville, J.E.; Mali, P.; Rios, X.; Aach, J.; Church, G.M. Genome engineering in Saccharomyces cerevisiae using CRISPR-Cas systems. Nucleic Acids Res. 2013, 41, 4336-4343. [CrossRef] 
31. Kondo, K.; Saito, T.; Kajiwara, S.; Takagi, M.; Misawa, N. A transformation system for the yeast Candida utilis: Use of a modified endogenous ribosomal protein gene as a drug-resistant marker and ribosomal DNA as an integration target for vector DNA. J. Bacteriol. 1995, 177, 7171-7177. [CrossRef]

32. Gupta, N.; Rathi, P.; Gupta, R. Simplified para-nitrophenyl palmitate assay for lipases and esterases. Analytical Biochem. 2002, 311, 98-99. [CrossRef]

33. Funk, I.; Rimmel, N.; Schorsch, C.; Sieber, V.; Schmid, J. Production of dodecanedioic acid via biotransformation of low cost plant-oil derivatives using Candida tropicalis. J. Ind. Microbiol. Biotechnol. 2017, 44, 1491-1502. [CrossRef]

34. Cao, Z.; Gao, H.; Liu, M.; Jiao, P. Engineering the acetyl-CoA transportation system of Candida tropicalis enhances the production of dicarboxylic acid. Biotechnol. J. 2006, 1, 68-74. [CrossRef]

35. Lee, H.; Han, C.; Lee, H.W.; Park, G.; Jeon, W.; Ahn, J.; Lee, H. Development of a promising microbial platform for the production of dicarboxylic acids from biorenewable resources. Biotechnol. Biofuels 2018, 11, 310. [CrossRef]

36. Cao, W.; Li, H.; Luo, J.; Yin, J.; Wan, Y. High-level productivity of $\alpha, \omega$-dodecanedioic acid with a newly isolated Candida viswanathii strain. J. Ind. Microbiol. Biotechnol. 2017, 44, 1191-1202. [CrossRef]

37. Schwartz, C.M.; Hussain, M.S.; Blenner, M.; Wheeldon, I. Synthetic RNA Polymerase III Promoters Facilitate High-Efficiency CRISPR-Cas9-Mediated Genome Editing in Yarrowia lipolytica. ACS Synth. Biol. 2016, 5, 356-359. [CrossRef]

38. Ryan, O.W.; Skerker, J.M.; Maurer, M.J.; Li, X.; Tsai, J.C.; Poddar, S.; Lee, M.E.; DeLoache, W.; Dueber, J.E.; Arkin, A.P.; et al. Selection of chromosomal DNA libraries using a multiplex CRISPR system. Elife 2014, 3, e03703. [CrossRef]

39. Mans, R.; van Rossum, H.M.; Wijsman, M.; Backx, A.; Kuijpers, N.G.; van den Broek, M.; Daran-Lapujade, P.; Pronk, J.T.; van Maris, A.J.; Daran, J.M.G. CRISPR/Cas9: A molecular Swiss army knife for simultaneous introduction of multiple genetic modifications in Saccharomyces cerevisiae. FEMS Yeast Res. 2015, 15, fov004. [CrossRef]

40. Arras, S.D.; Chua, S.M.; Wizrah, M.S.; Faint, J.A.; Yap, A.S.; Fraser, J.A. Targeted genome editing via CRISPR in the pathogen cryptococcus neoformans. PLoS ONE 2016, 11, e0164322. [CrossRef]

41. Kieliszek, M.; Kot, A.M.; Bzducha-Wróbel, A.; BŁażejak, S.; Gientka, I.; Kurcz, A. Biotechnological use of Candida yeasts in the food industry: A review. Fungal. Biol. Rev. 2017, 31, 185-198. [CrossRef]

42. Pfaller, M.A.; Castanheira, M.; Messer, S.A.; Moet, G.J.; Jones, R.N. Variation in Candida spp. distribution and antifungal resistance rates among bloodstream infection isolates by patient age: Report from the SENTRY Antimicrobial Surveillance Program (2008-2009). Diagn. Microbiol. Infect. Dis. 2010, 68, 278-283. [CrossRef] [PubMed]

43. Wang, H.J.; Le Dall, M.T.; Wach, Y.; Laroche, C.; Belin, J.M.; Gaillardin, C.; Nicaud, J.M. Evaluation of acyl coenzyme A oxidase (Aox) isozyme function in the n-alkane-assimilating yeast Yarrowia lipolytica. J. Bacteriol. 1999, 181, 5140-5148. [CrossRef] [PubMed]

44. Fu, Y.; Foden, J.A.; Khayter, C.; Maeder, M.L.; Reyon, D.; Joung, J.K.; Sander, J.D. High-frequency off-target mutagenesis induced by CRISPR-Cas nucleases in human cells. Nat. Biotechnol. 2013, 31, 822-826. [CrossRef] [PubMed]

45. Hsu, P.D.; Scott, D.A.; Weinstein, J.A.; Ran, F.A.; Konermann, S.; Agarwala, V.; Li, Y.; Fine, E.J.; Wu, X.; Shalem, O.; et al. DNA targeting specificity of RNA-guided Cas9 nucleases. Nat. Biotechnol. 2013, 31, 827-832. [CrossRef] [PubMed]

(C) 2020 by the authors. Licensee MDPI, Basel, Switzerland. This article is an open access article distributed under the terms and conditions of the Creative Commons Attribution (CC BY) license (http://creativecommons.org/licenses/by/4.0/). 